\title{
Changes in physical activity associated with the COVID-19 pandemic in individuals with overweight and obesity: an interrupted time series analysis with historical controls
}

\author{
Michael C. Robertson ${ }^{1,2} \cdot$ Che Young Lee ${ }^{1}$ Ivan Haw-Chong Wu ${ }^{3}$ Yue Liao ${ }^{4}$ Margaret Raber $^{1,5}$. \\ Nathan Parker ${ }^{6} \cdot$ Thuan Le $^{1} \cdot$ Leticia Gatus $^{7} \cdot$ Karen M. Basen-Engquist $^{1}$ iD
}

Received: 5 July 2021 / Accepted: 11 October 2021 / Published online: 26 October 2021

(C) The Author(s), under exclusive licence to Springer Science+Business Media, LLC, part of Springer Nature 2021

\begin{abstract}
The objective of this study was to investigate changes in physical activity patterns associated with the COVID-19 pandemic in individuals with overweight and obesity who were participating in a school district worksite weight loss program. We conducted comparative design interrupted time series analyses on physical activity device (Fitbit) data from the 2018-2019 and 2019-2020 school years $(\mathrm{N}=211)$. We administered a questionnaire in 2020 to supplement device data. After the stay-at-home orders in 2020, participants tended to decrease their weekly step count $(\mathrm{B}=-1315.7, \mathrm{SE}=627.7, p=.045)$, decrease their weekly "Lightly active minutes" ( $\mathrm{B}=-39.1, \mathrm{SE}=12.6, p=.007)$, and increase their weekly "Very active minutes" compared
\end{abstract}

Karen M. Basen-Engquist

kbasenen@mdanderson.org

Michael C. Robertson

mcrobert@utmb.edu

Che Young Lee

clee12@mdanderson.org

Ivan Haw-Chong Wu

ihwu@mdanderson.org

Yue Liao

yue.liao@uta.edu

Margaret Raber

margaret.raber@bcm.edu

Nathan Parker

nathan.parker@moffitt.org

Thuan Le

tale@mdanderson.org

Leticia Gatus

lagatus@mdanderson.org

1 Department of Behavioral Science, The University of Texas MD Anderson Cancer Center, 1515 Holcombe Boulevard, Houston, TX 77030, USA to their counterparts from the year before $(B=7.6, \mathrm{SE}=3.2$, $p=.020)$. Decreased motivation, gym closures, and safety concerns were cited as barriers to physical activity. Having more time and health consciousness were cited as facilitators of physical activity. The COVID-19 pandemic was related to changes in physical activity in both positive and negative ways, revealing opportunities to promote healthy lifestyle behaviors in this population. More research is needed to determine optimal approaches to health promotion in the post-COVID-19 era.

Keywords Physical activity · Obesity · COVID-19 . Interrupted time series analysis

2 Department of Nutrition, Metabolism \& Rehabilitation Sciences, The University of Texas Medical Branch at Galveston, 301 University Boulevard, Galveston, TX 77555, USA

3 Department of Health Disparities Research, The University of Texas MD Anderson Cancer Center, 1400 Pressler St., Houston, TX 77030, USA

4 Department of Kinesiology, College of Nursing and Health Innovation, The University of Texas at Arlington, $411 \mathrm{~S}$ Nedderman Dr, Arlington, TX 76010, USA

5 Department of Pediatrics, Baylor College of Medicine, 1 Baylor Plaza, Houston, TX 77030, USA

6 Department of Health Outcomes and Behavior, Moffitt Cancer Center, Tampa, FL, USA

7 Ambulatory Operations, The University of Texas MD Anderson Cancer Center, 1515 Holcomb Boulevard, Houston, TX 77030, USA 


\section{Introduction}

On January 31, 2020, the U.S. Department of Health and Human Services declared a public health emergency in response to the respiratory disease that would become known as the (SARS-CoV2) coronavirus disease (COVID19). In March and April 2020, most state governments issued stay-at-home orders to minimize the spread of the disease. State stay-at-home orders included mandatory quarantine for travelers, bans on large social gatherings, school closures and closures of non-essential businesses, reduced access to parks, and advisories to stay home (Gostin \& Wiley, 2020). The necessary initiatives required to minimize the spread of the COVID-19 disease have been associated with marked social and environmental changes and have impacted various health behaviors such as physical activity.

Physical inactivity is associated with all-cause mortality and adverse health outcomes including cardiovascular disease, stroke, hypertension, obesity, diabetes, some types of cancer, and severe COVID-19 outcomes (McKinney et al., 2016; Sallis et al., 2021; Warburton et al., 2006). The U.S. Department of Health and Human Services has put forth physical activity guidelines for Americans (US Department of Health and Human Services, 2018). Adherence to these recommended guidelines before the pandemic was suboptimal, with only $65.2 \%$ of Americans reporting meeting the aerobic physical activity guideline (Du et al., 2019). While necessary to curb the spread of the disease, stay-at-home initiatives may have led to changes in activity patterns (e.g., decreases in physical activity and increases in sedentary behavior). Researchers speculated that leisure-time physical activity might be reduced during the pandemic due to disruption of individuals' daily routines and widespread closure of exercise facilities (Hall et al., 2020); further, transportation-related and occupational physical activity may be supplanted by sedentary time due to stay-at-home initiatives and unemployment (Hall et al., 2020). Emerging self-reported (Dunton et al., 2020; Flanagan et al., 2020; Knell et al., 2020; Meyer et al., 2020) and device-measured data (Di Sebastiano et al., 2020; Ong et al., 2020) suggest that physical activity may have indeed decreased as a result of the stay-at-home orders.

Individuals with overweight and obesity may be particularly prone to the deleterious health consequences associated with physical inactivity. Physical inactivity can contribute to weight gain, and a recent study found that individuals with obesity reported gaining an average of $1.5 \mathrm{kgs}$ within one month of observing stay-at-home orders (Pellegrini et al., 2020). This is important because weight gained over a short period of time can lead to lasting changes in body weight (Schoeller, 2014). Further, decreases in physical activity may exacerbate poor psychological wellbeing (Carriedo et al., 2020; Duncan et al., 2020; Jiménez-Pavón et al., 2020;
Schuch et al., 2020), and further contribute to weight gain and other downstream physical health complications (Block et al., 2009). Home-based physical activity has been proposed as a preventive measure for weight gain during the COVID-19 pandemic (Bhutani \& Cooper, 2020), but to date limited research has investigated how physical activity may have changed in overweight and obese individuals during the pandemic.

The workplace is an important potential source of physical activity, and school districts are promising settings to implement behavioral interventions (Schultz et al., 2020). Occupational physical activity is negatively associated with obesity, however, teaching is a profession associated with relatively low levels of occupational physical activity (Church et al., 2011; King et al., 2001). From a public health perspective, teachers may be a particularly important population to serve because, in addition to constituting a large population, they can also act as role models to influence energy balance-related behaviors in their students and their well-being can have bearing on student achievement (Lambrinou et al., 2020; Schultz et al., 2020). Understanding changes in physical activity patterns of school district employees with overweight and obesity can help inform worksite interventions set within the context of a professional environment that has been altered by the COVID-19 pandemic.

The first aim of this study was to evaluate changes in device-measured physical activity patterns associated with COVID-19 in a sample of individuals with overweight and obesity participating in a school district's worksite weight loss program. Second, we aimed to explore participants' perceptions of reasons for changes in various domains of physical activity. We hypothesized that the stay-at-home orders stemming from the COVID-19 pandemic would be associated with immediate and sustained reductions in device-measured physical activity, and that participants would report decreases in leisure time, occupational, and transport-related physical activity.

\section{Methods}

\section{Intervention}

Vibrant Lives Plus was a six-month weight loss program for school district employees with overweight or obesity (body mass index $>=27 \mathrm{gk} / \mathrm{m}^{2}$ ) near Houston, Texas. It occurred over three cohorts (reflecting the 2017-2018, 2018-2019, and 2019-2020 school years). In the present study, we analyzed data from the final two cohorts only (we omitted the first cohort due to differences in its eligibility criteria and intervention content). Vibrant Lives Plus was a part of the Pasadena Vibrant Community, an initiative to unite 
individuals, schools, workplaces, and other key stakeholders to make lasting changes in health behaviors. The weight loss program was based on principles from the Diabetes Prevention Program. It featured 16 lessons that participants received by email and/or mail over the course of 26 weeks and was reinforced by 10 automated text messages sent each week. The first eight lessons were sent weekly, and the second eight lessons were sent every two weeks; text messages were sent each week for 26 weeks. Participants were encouraged to limit sedentary time and gradually increase their activity levels to achieve $150 \mathrm{~min}$ of moderate-to-vigorous intensity physical activity per week via low impact forms of exercise (not requiring exercise equipment). At the beginning of the program, participants also received a Fitbit Flex 2 (a wrist-worn physical activity tracker). Study staff assisted participants in downloading the Fitbit mobile app and instructed participants to regularly track their physical activity for the duration of the program. The University of Texas MD Anderson Cancer Center institutional review board approved the analysis of these data.

\section{Measures}

Each year, we administered surveys at baseline (November-December) and post-intervention (April-May) via Qualtrics survey software. Survey items gathered data on sociodemographic characteristics. For the 2019-2020 cohort, we added survey items pertaining to perceived behavioral impacts of the COVID-19 pandemic.

\section{Device-measured physical activity}

The Fitbit Flex 2 is a wrist-worn device that contains a 3 -axis accelerometer and can link wirelessly to mobile devices. Proprietary Fitbit algorithms use incoming data to characterize motion patterns. Output includes estimates of daily step count as well as minutes spent "very active", "fairly active", and "lightly active". We evaluated changes in each of these movement patterns independently. We summed daily data by week to align with nationally recommended physical activity guidelines and because week-level estimates may be more accurate than other ways of aggregating Fitbit data (Brewer et al., 2017). We defined a day of valid wear as a day in which the Fitbit device recorded at least 1500 steps (Chu et al., 2017; Tudor-Locke et al., 2015) or at least $10 \mathrm{~h}$ of valid wear according to the application of a standard protocol for ActiGraph wear time to minute-level data (i.e., greater than 60 consecutive minutes of 0 steps, with 2 a minute tolerance, was deemed nonwear) (Choi et al., 2011). Also in accordance with commonly used accelerometer and Fitbit data handling procedures, we defined a week of valid wear as a week in which participants had at least four valid wear days (Chu et al., 2017; Colley et al., 2010).

\section{Perceptions of changes in physical activity}

In the post-intervention survey for the 2019-2020 cohort, we included items to provide insight on how participants felt the COVID-19 pandemic impacted their physical activity patterns. The first such item stated,

"How has the COVID-19 pandemic affected your moderate- to vigorous-intensity aerobic physical activity (also known as cardio, examples include: brisk walking, jogging, bicycling or indoor cycling, swimming, or aerobic dancing)? This could also include occupation-related physical activity, transportation-related physical activity and household chores."

The four response options included 1) moderate- to vigorous-intensity aerobic physical activity (MVPA) has not changed, 2) the amount of MVPA is about the same, but the activities/exercise have changed, 3) MVPA increased, or 4) MVPA decreased. We included a similar item for muscle strengthening physical activity. If, in the item pertaining to MVPA, participants indicated anything other than that their MVPA has not changed, they additionally received questions asking whether their occupational, transportation-related, household-related, and leisure time physical activity had each (1) decreased, (2) stayed the same, or (3) increased. Participants who reported that their physical activity changed were also presented with a checklist of items to identify specific factors that may have caused the change (e.g., more time for exercise; less motivation; entertaining/ walking more with young children, etc.) and the opportunity to write in additional responses.

\section{Statistical analyses}

We analyzed data from the 2018-2019 and 2019-2020 cohorts of the Vibrant Lives Plus Program. We calculated descriptive statistics for participant characteristics and selfreported perceptions of changes in physical activity linked to the COVID-19 pandemic. We tested for differences between groups' sociodemographic profiles using Pearson's Chisquare test of independence.

For aim 1, we evaluated multilevel models in an interrupted time series design with a nonequivalent comparison group (Shadish et al., 2002). Interrupted time series is a type of trend analysis that uses piecewise regression to evaluate immediate (i.e., intercept) and gradual (i.e., slope) changes of in a dependent variable associated with a hypothesized interruption point (Shadish et al., 2002). For each cohort, we selected the fourth week of the new year as the start of the study period to avoid capturing aberrant physical activity patterns associated with the winter holidays. We selected the start of the 11th week of the year as the 
Table 1 Participant characteristics

\begin{tabular}{|c|c|c|c|c|}
\hline Characteristic & Category & $\begin{array}{l}2019 ; N=100 \\
\%(n)\end{array}$ & $\begin{array}{l}2020 ; N=111 \\
\%(n)\end{array}$ & P-value ${ }^{a}$ \\
\hline \multirow[t]{4}{*}{ Age } & $18-34$ & $24(24)$ & $17(19)$ & 0.37 \\
\hline & $35-49$ & $51(51)$ & $55(61)$ & \\
\hline & $50-64$ & $22(22)$ & $27(30)$ & \\
\hline & $65+$ & $3(3)$ & $1(1)$ & \\
\hline \multirow[t]{2}{*}{ Gender } & Male & $10(10)$ & $11(12)$ & 1.00 \\
\hline & Female & $90(90)$ & $89(99)$ & \\
\hline \multirow[t]{4}{*}{ Education level } & HS diploma/GED or less & $6(6)$ & $5(6)$ & 0.71 \\
\hline & Technical school or some college & $16(16)$ & $17(19)$ & \\
\hline & Bachelor's degree & $36(36)$ & $31(34)$ & \\
\hline & Graduate school & $42(42)$ & $47(52)$ & \\
\hline \multirow[t]{3}{*}{ Marital status } & Single & $19(19)$ & $22(24)$ & 0.34 \\
\hline & Married or living with significant other & $71(71)$ & $67(74)$ & \\
\hline & Divorced or separated & $10(10)$ & $12(13)$ & \\
\hline \multirow[t]{4}{*}{ Race/ethnicity } & Black or African American & $12(12)$ & $13(14)$ & 0.59 \\
\hline & Hispanic & $31(31)$ & $35(39)$ & \\
\hline & Non-Hispanic White & $52(52)$ & $47(52)$ & \\
\hline & Other & $5(5)$ & $5(6)$ & \\
\hline \multirow[t]{2}{*}{ Body mass index category } & Overweight & $17(17)$ & $25(28)$ & 0.17 \\
\hline & Obese & $83(83)$ & $73(81)$ & \\
\hline
\end{tabular}

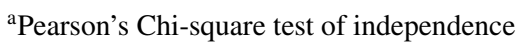

hypothesized interruption point because in both 2019 and 2020 this occurred during the week of spring break, and in 2020 employees did not return to the workplace for the rest of the school year due to state stay-at-home orders (employees were not allowed back in school buildings unless scheduled with the principal to get materials for home). Teachers taught from home starting March 23rd, 2020 (two weeks after spring break). We selected the 17 th week of the year as the end of the study period, as this was the last full week of data obtained in both years prior to the end of data collection. Texas stay-at-home orders were still in place at the start of this week (including public school closures), with some re-opening measures set to take effect in May 2020 (e.g., opening of in-store retail establishments and dine-in restaurants at up to $25 \%$ of the total listed occupancy) (Texas Department of State Health Services, 2021).

Multilevel models included terms for: (1) cohort (binary; 2019 [0] vs. 2020 [1]), (2) weeks (continuous; weeks over the whole study period [4-17]), (3) phase (binary; before [0] vs. after the interruption point [1]), (4) weeks after the interruption point, (5) weeks for 2020 group only, (6) phase for 2020 group only (this parameter is interpretable as the level change in the dependent variable associated with the COVID-19 pandemic), and (7) weeks after the interruption point for the 2020 group only (this parameter is interpretable as the slope change in the dependent variable associated with the COVID-19 pandemic). We specified random terms for time (weeks) nested within individuals. All models adjusted for weekly valid device wear (models did not adjust for sociodemographic factors; such variables typically remain fairly constant over the time frame involved in the present study and thus the risk of confounding is minimal (Bernal et al., 2017)). We investigated the autocorrelation error structure of these models via complete and partial auto-correlation function plots and used Bayesian Information Criteria to select among competing models with different error structure specifications. We found an AR-1 process to fit all models best, and for all final models we used restricted maximum likelihood estimation. We evaluated separate models for each outcome variable: average weekly step count, Fitbit "very active minutes", Fitbit "fairly active minutes", and Fitbit "lightly active minutes". We conducted multiple imputation to handle missing data $(\mathrm{m}=5)$ and present pooled results (Allison, 2001; Rubin, 1996). We set the nominal $\alpha$ value to 0.05 for all analyses, which we performed in $\mathrm{R}$ version 4.0.3. 
Fig. 1 Average daily step count by week for each cohort

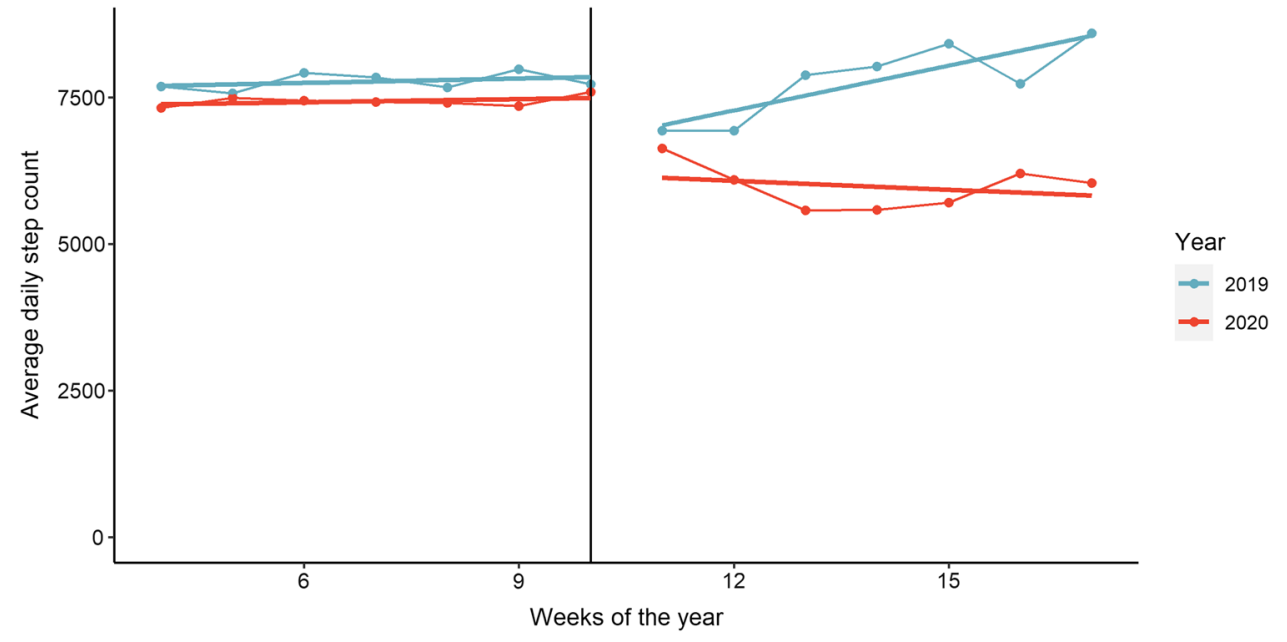

\section{Results}

\section{Participant characteristics}

Two hundred and eleven individuals participated in Vibrant Lives Plus and completed the baseline survey over the 2018-2019 and 2019-2020 school years. Participants were mostly female and were relatively well educated (Table 1). The mean age for 2019 was 42.6 years $(\mathrm{SD}=10.1)$, and that for 2020 was $43.2(\mathrm{SD}=10.0)$. Most participants had obesity at baseline (Table 1). The two cohorts did not have any significant differences in demographic variables.

\section{Comparative design interrupted time series analysis}

\section{Overall step count and fitbit lightly active minutes}

We did not observe evidence for an immediate, differential (level) change in step count by year after spring break $(p=0.357)$. We observed a statistically significant time trend (slope) such that after the hypothesized interruption point participants in 2020 tended to have a lower step count over time than their counterparts in $2019(B=-1315.7$, $\mathrm{SE}=627.7, \mathrm{df}=30.4, p=0.045$ ). Participants in 2019 appear to have increased their step count after an initial drop associated with spring break, whereas this was not the case in 2020 (Fig. 1).

Findings for Fitbit "lightly active minutes" mirrored those of overall step count. We did not observe evidence for an immediate, differential phase change in Fitbit "lightly active minutes" $(p=0.572)$. We did observe evidence of a statistically significant time trend such that after the hypothesized interruption point participants in 2020 tended to have a lower number of average Fitbit "lightly active minutes" over time than their counterparts in $2019(\mathrm{~B}=-39.1, \mathrm{SE}=12.6$, $\mathrm{df}=15.1, \mathrm{p}=0.007)$.
Fitbit fairly and very active minutes

We did not observe statistically significant differences in Fitbit "fairly active minutes" in either an immediate shift $(p=0.202)$ or time trends $(p=0.301)$ by year after the hypothesized interruption point. We did not observe evidence for a differential, immediate change in Fitbit "very active minutes" by year after spring break $(p=0.407)$. We observed a statistically significant time trend such that after the hypothesized interruption point participants in 2020 tended to register more minutes of Fitbit "very active minutes" over time than their counterparts in $2019(\mathrm{~B}=7.6$, $\mathrm{SE}=3.2, \mathrm{df}=219.4, p=0.020)$. Participants in 2020 appear to have increased their Fitbit "very active minutes" over time after spring break in 2020 (Fig. 2).

\section{Self-reported changes in physical activity-related factors}

Nearly half of participants (48\%) indicated that they reduced their overall amount of aerobic physical activity, and over a third of participants (37\%) indicated that they reduced their overall amount of muscle strengthening physical activity as a result of the COVID-19 pandemic (Fig. 3). Most participants indicated that they reduced their occupation-related and transport-related physical activity, and increased their household-related physical activity (Fig. 3). Participants were approximately evenly split with respect to leisure-time physical activity, with 39\% reporting increases and $40 \%$ reporting decreases (Fig. 3).

\section{Aerobic PA}

Participants identified lack of motivation (37\% endorsing this barrier), facilities being closed (31\%), and potential lack of adequate social distancing (27\%) to be primary barriers to 
Fig. 2 Average daily Fitbit very active minutes by week for each cohort

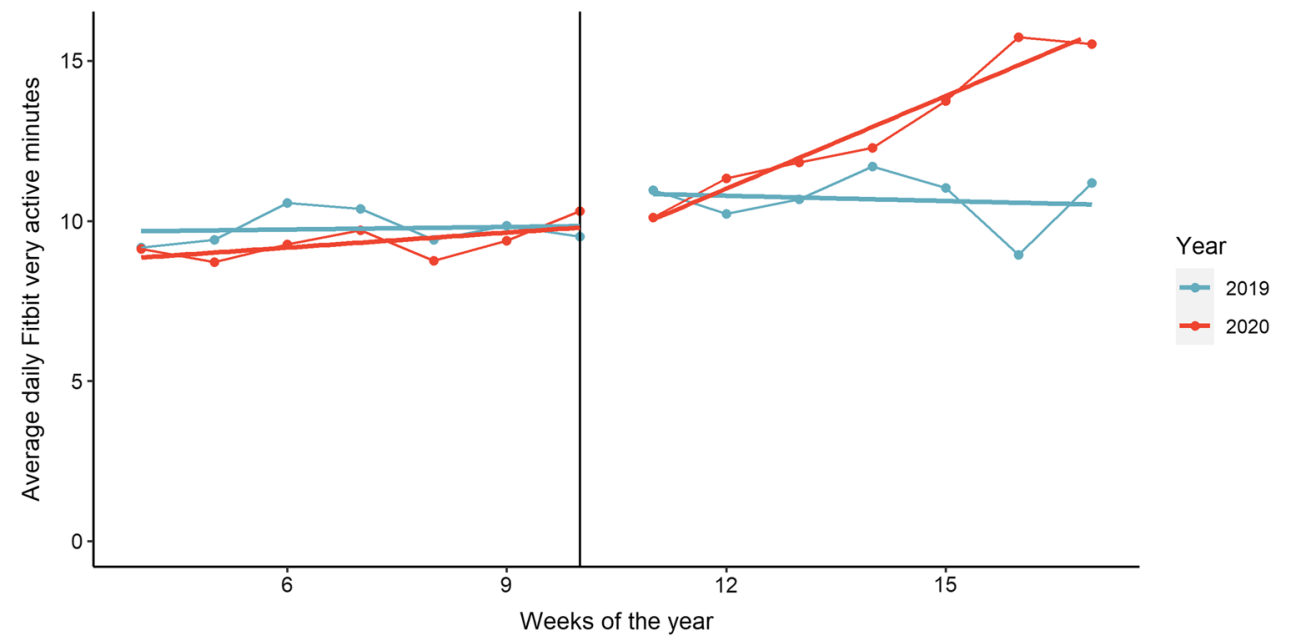

Overall changes in self-reported physical activity

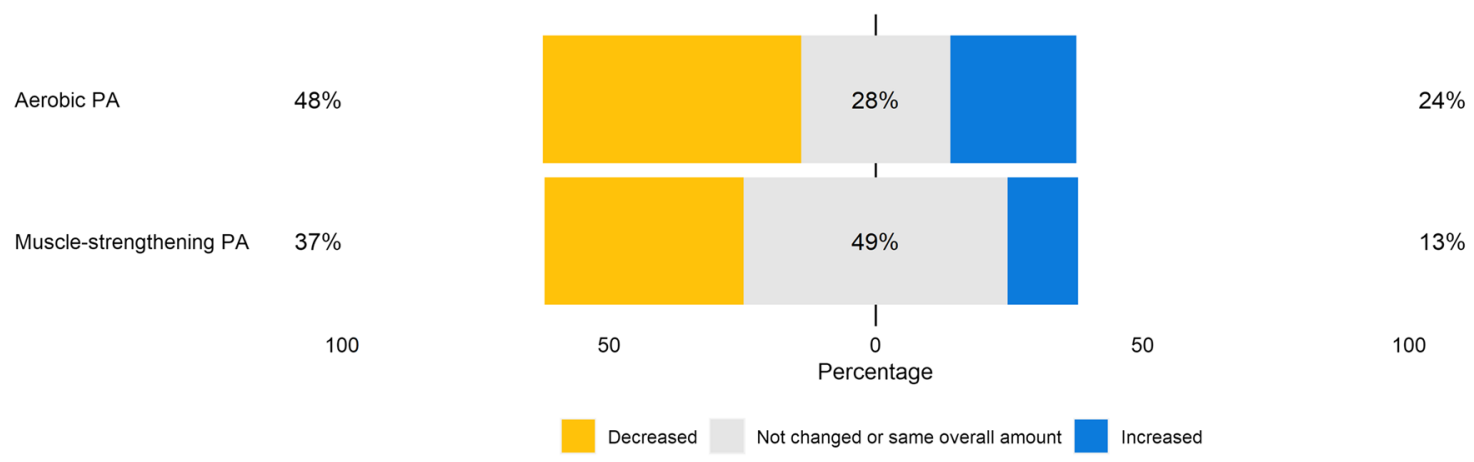

Changes in domain-specific physical activity

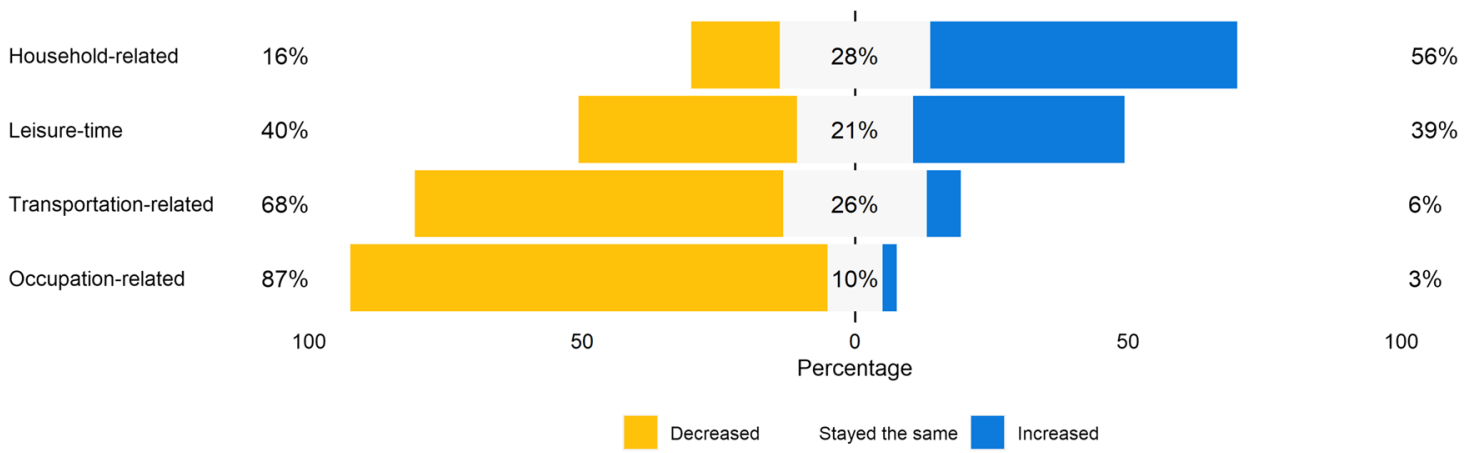

Fig. 3 Perceived changes in physical activity because of the COVID-19 pandemic

physical activity during this time (Fig. 4). Having more time for exercise (31\%) and trying to get fitter to protect health (28\%) emerged as facilitators to physical activity (Fig. 4). Experiencing a personal injury and caregiving for a special needs person were two additional responses that participants indicated had impacted their physical activity levels.

\section{Discussion}

Results indicate that a sample of school district employees participating in a worksite weight loss program tended to decrease step count and Fitbit "lightly active minutes" and increase Fitbit "very active minutes" in association with the enactment of state stay-at-home orders. Participants reported a perceived decrease in occupation- and transport-related 
Barriers to physical activity

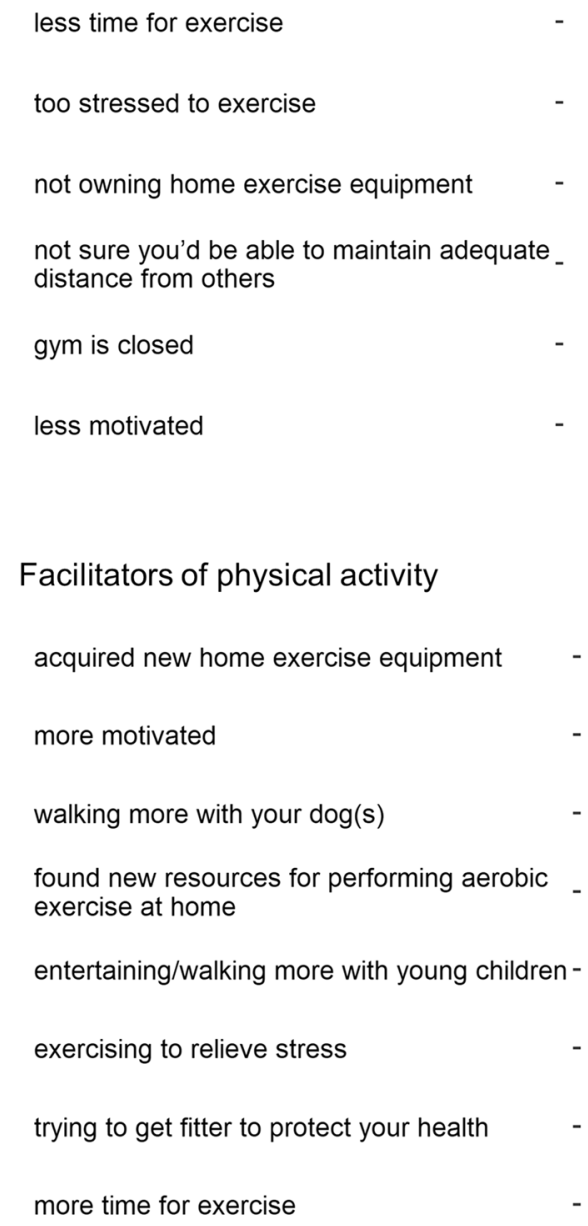

Facilitators of physical activity

acquired new home exercise equipment

more motivated

walking more with your dog(s)

found new resources for performing aerobic exercise at home

entertaining/walking more with young children -

exercising to relieve stress

trying to get fitter to protect your health

more time for exercise
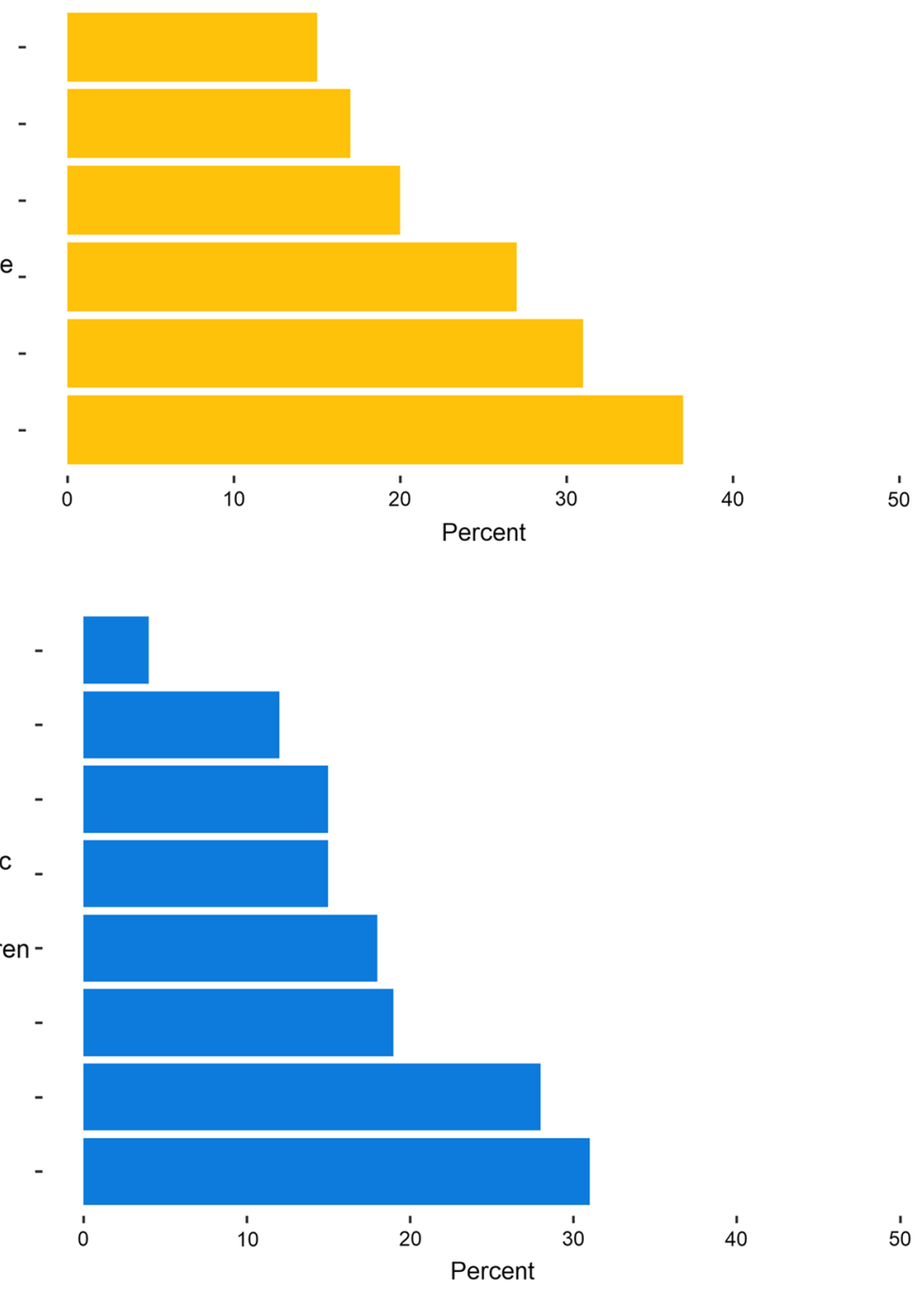

Fig. 4 Perceived barriers and facilitators of physical activity

physical activity, and an increase in household-related physical activity. Participants reported increases and decreases in leisure-time physical activity in approximately equal numbers, and cited lacking motivation, gym closures, and pandemic-related safety concerns as primary barriers to physical activity. Having more time to engage in physical activity and health consciousness were most endorsed as facilitators of physical activity.

The finding that daily step count and Fitbit "lightly active minutes" decreased is in accord with existing literature on the COVID-19 pandemic (Cheval et al., 2020; Di Sebastiano et al., 2020; Dunton et al., 2020; Knell et al., 2020; LópezBueno et al., 2020; Ong et al., 2020; Rhodes et al., 2020). This has important public implications because it points to an alarming trend in which individuals have decreased light-intensity physical activity and increased sedentary time as a result of the pandemic. As sedentary behavior is associated with premature mortality, obesity, type 2 diabetes and cardiovascular disease (Dunstan et al., 2012; Hamilton et al., 2007; Owen et al., 2010), it is important to continue to investigate the downstream behavioral and health related impacts of the COVID-19 pandemic. Further, it is important that surveillance efforts are in place to understand to what degree deleterious, population-level changes in physical activity patterns persist.

The finding indicating that our sample tended to increase Fitbit "very active minutes" was not expected. While some research has found increases in walking and moderate-intensity physical activity to be associated with the pandemic (Cheval et al., 2020), most studies suggest that the COVID19 pandemic was associated with decreases in moderate-tovigorous physical activity (Di Sebastiano et al., 2020; Dunton et al., 2020; Knell et al., 2020; Meyer et al., 2020; Ong et al., 2020). Vibrant Lives participants had characteristics, 
including full time employment, relatively young age, and being relatively well-educated, that may have been healthprotective in the context of maintaining physical activity through the disruptive conditions of the pandemic (Knell et al., 2020; López-Bueno et al., 2020; Rhodes et al., 2020). It is also possible that participants leveraged skills taught in the ongoing intervention to make positive changes during this time. Further, it is possible that federal and local approaches to navigating the pandemic had bearing on why results from this study in south Texas differed from research conducted in other parts of the world. Taken together, our results align somewhat with findings from Meyer et al. (2020) that found particularly high levels of both sedentary behavior and leisure time physical activity in a large convenience sample in April 2020 (Meyer et al., 2020). Meyer and colleagues offered as a potential explanation that individuals in the sample may have tended to engage in physical activity during this period for socialization purposes and/or in lieu of commuting to work.

Self-report data in the present study provided insight as to how physical activity patterns may have changed. Findings indicating perceived decreases in work- and transport-related physical activity align with findings from other research (Cheval et al., 2020; Meyer et al., 2020). While most participants in the present study reported an increase in their household-related physical activity, it appears that any such increases did not match decreases in other domains of physical activity given the overall decrease in step count and Fitbit "lightly active minutes". Findings for leisure time physical activity were split, with about $40 \%$ of the sample reporting an increase, and about an equal percentage reporting a decrease. This is particularly important, because leisure-time physical activity is most directly linked to achieving moderate-to-vigorous levels of intensity and meeting nationally recommended physical activity guidelines (US Department of Health and Human Services., 2018).

Findings indicate that decreased motivation and concerns about possible exposure to the virus that causes COVID-19 were important barriers to engaging in physical activity, and that engaging in physical activity to reduce some of the risks associated with COVID-19 was a motivator for engaging in physical activity. These findings align with other research that has highlighted the role that health consciousness seems to have played in maintaining physical activity through the pandemic (Pu et al., 2020). Perceived threat from COVID-19 may have impelled some participants to engage in healthy lifestyle behaviors.

Early research encouraged individuals with obesity to lose weight and engage in mild-to-moderate intensity physical activity to strengthen the immune system to protect against COVID-19 (Luzi \& Radaelli, 2020). Indeed, more recent evidence suggests that engaging in physical activity may be a protective factor against severe COVID-19 complications (Sallis et al., 2021). In light of our finding that the most commonly endorsed barrier to physical activity was a decreased motivation, highlighting that physical activity can serve as a potent non-pharmacological immunomodulatory intervention may be a useful point for public health messaging (while continuing to emphasize the importance of social distancing, the appropriate use of personal protective equipment, and the safety and efficacy of vaccination).

Findings also revealed that gym closures and not owning home exercise equipment were commonly cited barriers to engaging in physical activity. This aligns with other research that highlights the importance of home-based exercise equipment in maintaining physical activity through the study period (Dunton et al., 2020; Kaushal et al., 2020; Rhodes et al., 2020). Having more time to engage in physical activity was commonly cited as a facilitator of physical activity, and having physical activity equipment and/or access to online/ streaming classes with more discretionary time may have facilitated more vigorous-intensity physical activity (Dunton et al., 2020). These may present opportunities for behavioral intervention.

Many changes to the work environment wrought by the COVID-19 pandemic may persist indefinitely, including an openness from employers to working from home. Indeed, there is now a considerable proportion of the U.S. workforce that now has a more flexible off-site work schedule (Bureau, n.d.). Results from the present study suggest that these changing circumstances may affect physical activity patterns in both positive and negative ways. Decreased commuting time and the acquisition of resources for home-based exercise may facilitate more leisure-time, vigorous-intensity physical activity. It is also possible that decreases in active transport and occupational physical activity may tend to yield net increases in sedentary behavior and decreases in total physical activity volume. Workplace health and wellness programs will need to adapt to changing norms by leveraging new opportunities for health promotion and addressing emerging threats to employee health and wellness. More research is needed to determine optimal approaches to worksite physical activity programs in the post-COVID-19 era.

A limitation of this study is the threat of differential study attrition. The COVID-19 pandemic reduced motivation and ability to participate in scientific studies (Cardel et al., 2020). In this study, the percentage of weeks with valid Fitbit wear was between 65 and $67 \%$ for both cohorts before the hypothesized interruption point; after the hypothesized interruption point this percentage was 54\% in 2019 and $44 \%$ in 2020 . It may have been that those who ceased participating in the study in 2020 differed from those who ceased participating in 2019 in ways that violated the assumptions of multiple imputation. Another limitation of this study concerns its limited generalizability. This sample was a convenience sample from one specific geographic region that was predominantly 
female, relatively well educated, and may have been particularly motivated to engage in health-related lifestyle changes by virtue of participating in a worksite weight loss program. Strengths of this study include an ethnically diverse study sample, the parsing of device-measured, longitudinal data to obtain a relatively nuanced picture of physical activity patterns, the use of both objective and self-reported measures to triangulate changes in physical activity, the use of an interrupted time series study design that can provide strong evidence for causal inference (Shadish et al., 2002), and the use of a relatively robust method for handling missing data.

In conclusion, the COVID-19 pandemic appears to have led to changes in physical activity patterns in this sample of individuals with overweight and obesity participating in a school district's worksite weight loss program. While trends for a reduction in step count and Fitbit "lightly active minutes" are concerning, we also observed an encouraging trend for increase in Fitbit "very active minutes". Particularly given that many changes wrought by the COVID-19 pandemic may persist, it is important that researchers continue to investigate the changing landscape of determinants of health and wellness and emerging opportunities and barriers for behavioral intervention.

Acknowledgements This study was supported by the Center for Energy Balance in Cancer Prevention and Survivorship.

Authors' contributions M.C.R. and K.B-E conceived the project. M.C.R. and C.Y.L. conducted the statistical analyses. M.C.R. wrote the manuscript with support from C.Y.L., I.W., Y.L., M.R.R., N.P., T.L., L.G., and K.B.-E. T.L. and L.G. participated in data collection. All authors approved of the final version of this paper.

Funding Michael Robertson was supported by the National Cancer Institute of the National Institutes of Health (F31 CA236433). This study used MD Anderson's Assessment, Intervention, and Measurement (AIM) Core, a shared resource supported by the National Institutes of Health through MD Anderson's Cancer Center Support Grant (P30 CA016672). The content of this paper is solely the responsibility of the authors and does not necessarily represent the official views of the National Institutes of Health.

\section{Declarations}

Conflict of interest The authors declare that the research was conducted in the absence of any commercial or financial relationships that could be construed as a potential conflict of interest.

Code availability The code that support the findings of this study are available from the corresponding author, K.B.-E., upon reasonable request.

Consent to participate All participants consented to participate in this research
Ethics approval The analysis of these data was approved by the Institutional Review Board of the University of Texas MD Anderson Cancer Center

Informed consent Informed consent was obtained from all participants in the study.

Human and animal rights The procedures conducted in this study were in accordance with the ethical standards of the Institutional Review Board of the University of Texas MD Anderson Cancer Center.

\section{References}

Allison, P. D. (2001). Missing data. Sage publications.

Bernal, J. L., Cummins, S., \& Gasparrini, A. (2017). Interrupted time series regression for the evaluation of public health interventions: A tutorial. International Journal of Epidemiology, 46(1), 348-355. https://doi.org/10.1093/ije/dyw098

Bhutani, S., \& Cooper, J. A. (2020). COVID-19-Related Home Confinement in Adults: Weight Gain Risks and Opportunities. Obesity (silver Spring, Md.), 28(9), 1576-1577.

Block, J. P., He, Y., Zaslavsky, A. M., Ding, L., \& Ayanian, J. Z. (2009). Psychosocial stress and change in weight among US adults. American Journal of Epidemiology, 170(2), 181-192. https://doi.org/10.1093/aje/kwp104

Brewer, W., Swanson, B. T., \& Ortiz, A. (2017). Validity of Fitbit's active minutes as compared with a research-grade accelerometer and self-reported measures. BMJ Open Sport \& Exercise Medicine, 3(1), e000254.

Bureau, U. C. (n.d.). Working From Home During the Pandemic. The United States Census Bureau. Retrieved June 18, 2021, from https://www.census.gov/library/stories/2021/03/working-fromhome-during-the-pandemic.html

Cardel, M. I., Manasse, S., Krukowski, R. A., Ross, K., Shakour, R., Miller, D. R., Lemas, D. J., \& Hong, Y.-R. (2020). COVID-19 impacts mental health outcomes and ability/desire to participate in research among current research participants. Obesity, 28(12), 2272-2281.

Carriedo, A., Cecchini, J. A., Fernandez-Rio, J., \& Méndez-Giménez, A. (2020). COVID-19, psychological well-being and physical activity levels in older adults during the nationwide lockdown in Spain. The American Journal of Geriatric Psychiatry, 28(11), 1146-1155. https://doi.org/10.1016/j.jagp.2020.08.007

Cheval, B., Sivaramakrishnan, H., Maltagliati, S., Fessler, L., Forestier, C., Sarrazin, P., Orsholits, D., Chalabaev, A., Sander, D., \& Ntoumanis, N. (2020). Relationships between changes in selfreported physical activity, sedentary behaviour and health during the coronavirus (COVID-19) pandemic in France and Switzerland. Journal of Sports Sciences, 1-6.

Choi, L., Liu, Z., Matthews, C. E., \& Buchowski, M. S. (2011). Validation of accelerometer wear and nonwear time classification algorithm. Medicine and Science in Sports and Exercise, 43(2), 357-364. https://doi.org/10.1249/MSS.0b013e3181ed61a3

Chu, A. H., Ng, S. H., Paknezhad, M., Gauterin, A., Koh, D., Brown, M. S., \& Müller-Riemenschneider, F. (2017). Comparison of wrist-worn Fitbit Flex and waist-worn ActiGraph for measuring steps in free-living adults. PLOS ONE, 12(2), e0172535.

Church, T. S., Thomas, D. M., Tudor-Locke, C., Katzmarzyk, P. T., Earnest, C. P., Rodarte, R. Q., Martin, C. K., Blair, S. N., \& Bouchard, C. (2011). Trends over 5 decades in U.S. occupationrelated physical activity and their associations with obesity. PLoS ONE, 6(5), e19657. 
Colley, R., Gorber, S. C., \& Tremblay, M. S. (2010). Quality control and data reduction procedures for accelerometry-derived measures of physical activity. Health Reports, 21(1), 63.

Di Sebastiano, K. M., Chulak-Bozzer, T., Vanderloo, L. M., \& Faulkner, G. (2020). Don't walk so close to me: Physical distancing and adult physical activity in Canada. Frontiers in Psychology. https://doi.org/10.3389/fpsyg.2020.01895

Du, Y., Liu, B., Sun, Y., Snetselaar, L. G., Wallace, R. B., \& Bao, W. (2019). Trends in adherence to the physical activity guidelines for Americans for aerobic activity and time spent on sedentary behavior among US adults, 2007 to 2016. JAMA Network Open, 2(7), e197597-e197597.

Duncan, G. E., Avery, A. R., Seto, E., \& Tsang, S. (2020). Perceived change in physical activity levels and mental health during COVID-19: Findings among adult twin pairs. PLOS ONE, 15(8), e0237695. https://doi.org/10.1371/journal.pone.0237695

Dunstan, D. W., Howard, B., Healy, G. N., \& Owen, N. (2012). Too much sitting - A health hazard. Diabetes Research and Clinical Practice, 97(3), 368-376. https://doi.org/10.1016/j.diabres. 2012.05.020

Dunton, G. F., Wang, S. D., Do, B., \& Courtney, J. (2020). Early effects of the COVID-19 pandemic on physical activity locations and behaviors in adults living in the United States. Preventive Medicine Reports, 20, 101241. https://doi.org/10.1016/j. pmedr.2020.101241

Flanagan, E. W., Beyl, R. A., Fearnbach, S. N., Altazan, A. D., Martin, C. K., \& Redman, L. M. (2020). The impact of COVID-19 stay-at-home orders on health behaviors in adults. Obesity (silver Spring, Md.). https://doi.org/10.1002/oby.23066

Gostin, L. O., \& Wiley, L. F. (2020). Governmental public health powers during the COVID-19 pandemic: Stay-at-home orders, business closures, and travel restrictions. The Journal of the American Medical Association, 323(21), 2137-2138.

Hall, G., Laddu, D. R., Phillips, S. A., Lavie, C. J., \& Arena, R. (2020). A tale of two pandemics: How will COVID-19 and global trends in physical inactivity and sedentary behavior affect one another? Progress in Cardiovascular Diseases.

Hamilton, M. T., Hamilton, D. G., \& Zderic, T. W. (2007). Role of low energy expenditure and sitting in obesity, metabolic syndrome, Type 2 diabetes, and cardiovascular disease. Diabetes, 56(11), 2655-2667. https://doi.org/10.2337/db07-0882

Jiménez-Pavón, D., Carbonell-Baeza, A., \& Lavie, C. J. (2020). Physical exercise as therapy to fight against the mental and physical consequences of COVID-19 quarantine: Special focus in older people. Progress in Cardiovascular Diseases, 63(3), 386-388. https://doi.org/10.1016/j.pcad.2020.03.009

Kaushal, N., Keith, N., Aguiñaga, S., \& Hagger, M. S. (2020). Social cognition and socioecological predictors of home-based physical activity intentions, planning, and habits during the COVID19 pandemic. Behavioral Sciences, 10(9), 133. https://doi.org/ $10.3390 / \mathrm{bs} 10090133$

King, G. A., Fitzhugh, E. C., Jr., \& D. B., McLaughlin, J. E., Strath, S. J., Swartz, A. M., \& Thompson, D. L. (2001). Relationship of leisure-time physical activity and occupational activity to the prevalence of obesity. International Journal of Obesity, 25(5), 606.

Knell, G., Robertson, M. C., Dooley, E. E., Burford, K., \& Mendez, K. S. (2020). Health behavior changes during COVID-19 pandemic and subsequent "Stay-at-Home" orders. International Journal of Environmental Research and Public Health, 17(17), 6268. https://doi.org/10.3390/ijerph17176268

Lambrinou, C.-P., Androutsos, O., Karaglani, E., Cardon, G., Huys, N., Wikström, K., Kivelä, J., Ko, W., Karuranga, E., Tsochev, K., Iotova, V., Dimova, R., De Miguel-Etayo, P., González-Gil, M., \& E., Tamás, H., JANCSÓ, Z., Liatis, S., Makrilakis, K., \& Manios, Y. (2020). Effective strategies for childhood obesity prevention via school based, family involved interventions: A critical review for the development of the Feel4Diabetes-study school based component. BMC Endocrine Disorders, 20(Suppl 2), 52. https://doi.org/10.1186/s12902-020-0526-5

López-Bueno, R., Calatayud, J., Ezzatvar, Y., Casajús, J. A., Smith, L., Andersen, L. L., \& Lopez-Sanchez, G. F. (2020). Association between current physical activity and current perceived anxiety and mood in the initial phase of COVID-19 confinement. Frontiers in Psychiatry, 11.

Luzi, L., \& Radaelli, M. G. (2020). Influenza and obesity: Its odd relationship and the lessons for COVID-19 pandemic. Acta Diabetologica. https://doi.org/10.1007/s00592-020-01522-8

McKinney, J., Lithwick, D. J., Morrison, B. N., Nazzari, H., Isserow, S. H., Heilbron, B., \& Krahn, A. D. (2016). The health benefits of physical activity and cardiorespiratory fitness. British Columbia Medical Journal, 58(3), 131-137.

Meyer, J., McDowell, C., Lansing, J., Brower, C., Smith, L., Tully, M., \& Herring, M. (2020). Changes in physical activity and sedentary behaviour due to the COVID-19 outbreak and associations with mental health in 3,052 US adults. https://doi.org/ 10.33774/coe-2020-h0b8g

Ong, J. L., Lau, T., Massar, S. A. A., Chong, Z. T., Ng, B. K. L., Koek, D., Zhao, W., Yeo, B. T. T., Cheong, K., \& Chee, M. W. L. (2020). COVID-19-related mobility reduction: Heterogenous effects on sleep and physical activity rhythms. Sleep. https://doi. org/10.1093/sleep/zsaa179

Owen, N., Healy, G. N., Matthews, C. E., \& Dunstan, D. W. (2010). Too much sitting: The population-health science of sedentary behavior. Exercise and Sport Sciences Reviews, 38(3), 105-113. https://doi.org/10.1097/JES.0b013e3181e373a2

Pellegrini, M., Ponzo, V., Rosato, R., Scumaci, E., Goitre, I., Benso, A., Belcastro, S., Crespi, C., De Michieli, F., Ghigo, E., Broglio, F., \& Bo, S. (2020). Changes in weight and nutritional habits in adults with obesity during the "Lockdown" period caused by the COVID-19 virus emergency. Nutrients. https://doi.org/10. 3390/nu12072016

Pu, B., Zhang, L., Tang, Z., \& Qiu, Y. (2020). The relationship between health consciousness and home-based exercise in China during the COVID-19 pandemic. International Journal of Environmental Research and Public Health, 17(16), 5693. https:// doi.org/10.3390/ijerph17165693

Rhodes, R. E., Liu, S., Lithopoulos, A., Zhang, C.-Q., \& GarciaBarrera, M. A. (2020). Correlates of perceived physical activity transitions during the COVID-19 pandemic among canadian adults. Applied Psychology: Health and Well-Being, 12(4), $1157-1182$

Rubin, D. B. (1996). Multiple imputation after 18+ years. Journal of the American Statistical Association, 91(434), 473-489.

Sallis, R., Young, D. R., Tartof, S. Y., Sallis, J. F., Sall, J., Li, Q., Smith, G. N., \& Cohen, D. A. (2021). Physical inactivity is associated with a higher risk for severe COVID-19 outcomes: A study in 48440 adult patients. British Journal of Sports Medicine.

Schoeller, D. A. (2014). The effect of holiday weight gain on body weight. Physiology \& Behavior, 134, 66-69. https://doi.org/10. 1016/j.physbeh.2014.03.018

Schuch, F. B., Bulzing, R. A., Meyer, J., Vancampfort, D., Firth, J., Stubbs, B., Grabovac, I., Willeit, P., Tavares, V. D. O., Calegaro, V. C., Deenik, J., López-Sánchez, G. F., Veronese, N., Caperchione, C. M., Sadarangani, K. P., Abufaraj, M., Tully, M. A., \& Smith, L. (2020). Associations of moderate to vigorous physical activity and sedentary behavior with depressive and anxiety symptoms in self-isolating people during the COVID-19 pandemic: A cross-sectional survey in Brazil. Psychiatry Research, 292, 113339. https://doi.org/10.1016/j.psychres.2020.113339 
Schultz, N. S., Chui, K. K., Economos, C. D., Lichtenstein, A. H., Volpe, S. L., \& Sacheck, J. M. (2020). Impact of physical activity, diet quality and stress on cardiometabolic health in school employees. Preventive Medicine Reports, 20, 101243.

Shadish, W. R., Cook, T. D., \& Campbell, D. T. (2002). Experimental and quasi-experimental designs for generalized causal inference/William R. Shedish, Thomas D. Cook, Donald T. Campbell. Boston: Houghton Mifflin,.

Texas Department of State Health Services. (2021). Texas Executive Orders \& Public Health Disaster Declarations. Coronovirus Disease 2019. https://www.dshs.texas.gov/coronavirus/execo rders.aspx

Tudor-Locke, C., Barreira, T. V., \& Jr, J. M. S. (2015). Comparison of step outputs for waist and wrist accelerometer attachment sites. Medicine and Science in Sports and Exercise, 47(4), 839842. https://doi.org/10.1249/MSS.0000000000000476

US Department of Health and Human Services. (2018). Physical Activity Guidelines for Americans. 2nd ed. Washington, DC.

Warburton, D. E., Nicol, C. W., \& Bredin, S. S. (2006). Health benefits of physical activity: The evidence. CMAJ, 174(6), 801-809.

Publisher's Note Springer Nature remains neutral with regard to jurisdictional claims in published maps and institutional affiliations. 